\title{
Improvement of glass transition and flowability of reduced-fat coffee creamer: effect of fat replacer and fluidized bed drying
}

\begin{abstract}
This study was conducted to investigate the effects of inulin $(0,2.5,5$, and $7.5 \%)$, maltodextrin $(0,15,20$, and $25 \%)$, and different drying processes (one- and two-stage drying) on the morphology and physicochemical properties of regular and instant reduced-fat creamers. The present study showed that the drum-dried creamer containing $0 \%$ maltodextrin and $0 \%$ inulin was fully sticky powder with dark brown color. It was found that the maximum increase in maltodextrin (from 0 to $25 \%$ ) and inulin (from 0 to $7.5 \%$ ) resulted in the creamer with the highest glass transition temperature and the lowest stickiness among all formulated creamers. The application of two-stage drying involving fluidized bed drying resulted in further improvement of the glass transition temperature and stickiness of the reduced fat instant creamer. The instant creamers obtained from two-stage drying had considerably higher glass transition temperature and lower bulk density than the regular creamers from one-stage drying. Such improvement could be due to the reduction of bulk density induced by fluidized bed drying. This might be because of higher porosity of the creamer particles after agglomeration. The current study revealed that the addition of high amounts of inulin and maltodextrin also played a significant role in the reduction of bulk density and further improvement of glass transition temperature $(\mathrm{Tg})$ and solubility of the reduced fat creamer. The instant reduced fat creamer containing $25 \%$ maltodextrin and $7.5 \%$ inulin had the most desirable characteristics among all formulated creamers.
\end{abstract}

Keyword: Drum drying; Fluidized bed drying; Glass transition temperature (Tg); Inulin; Maltodextrin; Reduced-fat coffee creamer 